\title{
A rare cause of ileus: Gallstone ileus
}

\section{İleusun nadir bir nedeni: Safra taşı ileusu}

\author{
Meriç Emre Bostanci ${ }^{1}$, Onur Aveı ${ }^{2}$, Mustafa Atabey ${ }^{3}$
}

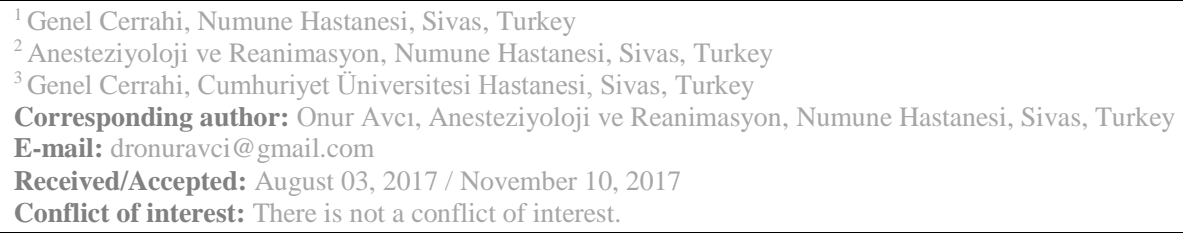

\section{Dear Editor}

Gallstone ileus occurs, when gallstones pass to the intestinal system through fistulas between bile ducts or the gallbladder and duodenum, stomach and colon, then cause an obstruction ${ }^{1}$. Biliaryenteric fistulas commonly form as a result of recurrent cholecystitis ${ }^{2}$. Intestinal obstruction occurs if the gallstone is $2.5 \mathrm{~cm}$ or bigger ${ }^{3}$. Obstructions commonly occur in terminal ileum $(70 \%)$, and less often in proximal ileum, jejunum, colon and duodenum ${ }^{4}$. Localizations of fistulas according to occurrence rate are cholecystoduodenal (76\%), cholecystocolic (11\%), cholecystogastric $(6 \%)$, choledochoduodenal $(4 \%)$, cholecystocholedochal (3\%); and gallstone ileus is seen in $0.3-0.5 \%$ of all cholelithiasis cases ${ }^{5}$.

76 year old female patient was admitted to the emergency service with right upper quadrant pain, nausea, vomiting and constipation for the last three days. She stated, that she had gallbladder stone for 10 years, and physical examination yielded Murphy's sign was positive. White blood count was detected as 12.000 in hemogram. ALT and AST levels were slightly elevated in liver function tests. The patient also had abdominal distension, and intestinal air-fluid levels were observed in her erect abdominal radiograph (Figure 1). In ultrasonography; the gallbladder appeared hydropic, wall thickness had a diffuse increase ( 9 $\mathrm{mm}$ on average), and there were stones in the lumen, with the biggest measured at $2.5 \mathrm{~cm}$. No dilatation was observed in extrahepatic and intrahepatic bile ducts. In computer tomography examination, an approximately $4 \times 3 \mathrm{~cm}$ sized round stone was detected at ileal segmental level, which filled the lumen entirely (Figure 2) and dilatation was observed at intestinal segments proximal of the stone. 


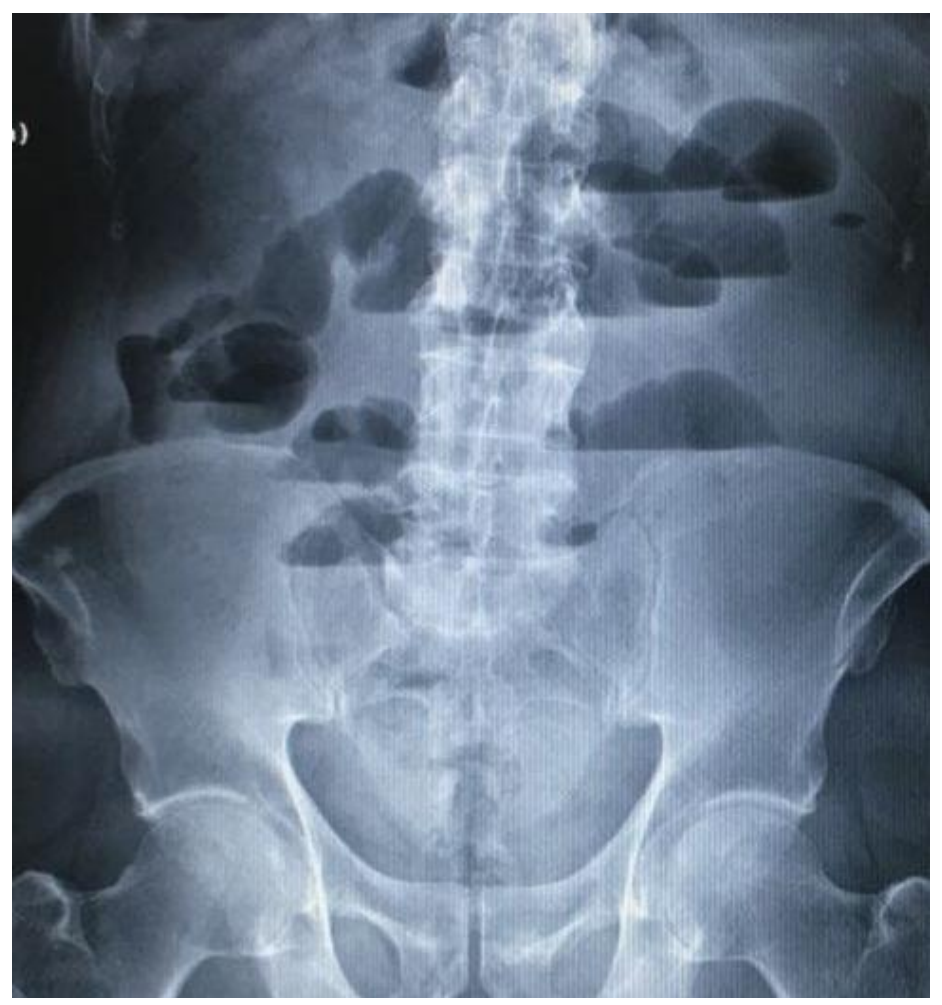

Figure 1. Abdominal distension and air-fluid levels.

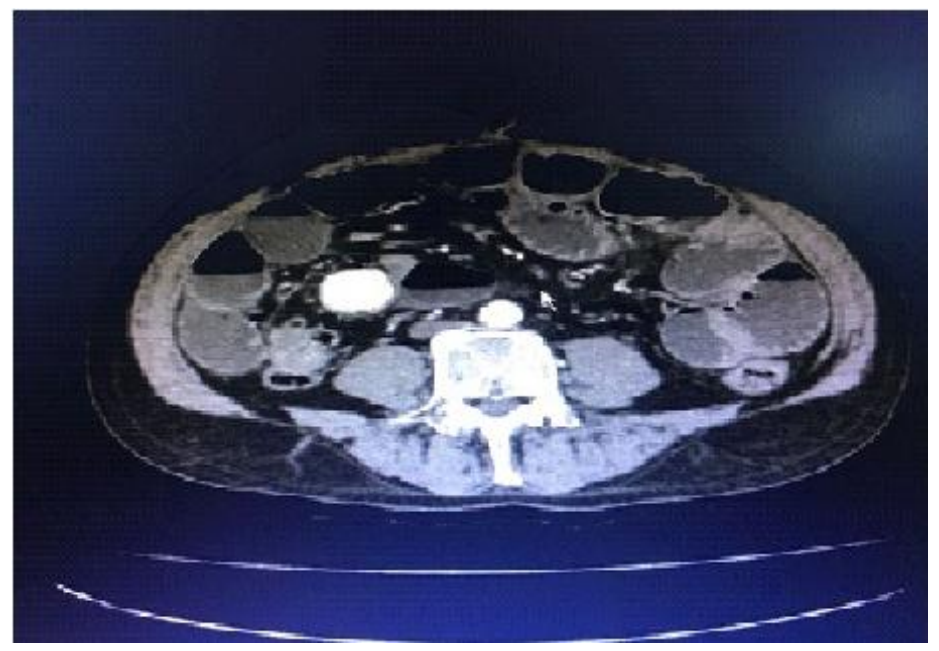

Figure 2. Round stone at ileal segment level

The patient was operated with gallstone ileus diagnosis. In the operation; starting from the Treitz ligament, the intestines were dilated and edematic till $150 \mathrm{~cm}$ distal of that point, and an approximately $4 \mathrm{~cm}$ big stone was seen inside the intestinal lumen at this level (Figure 3). 


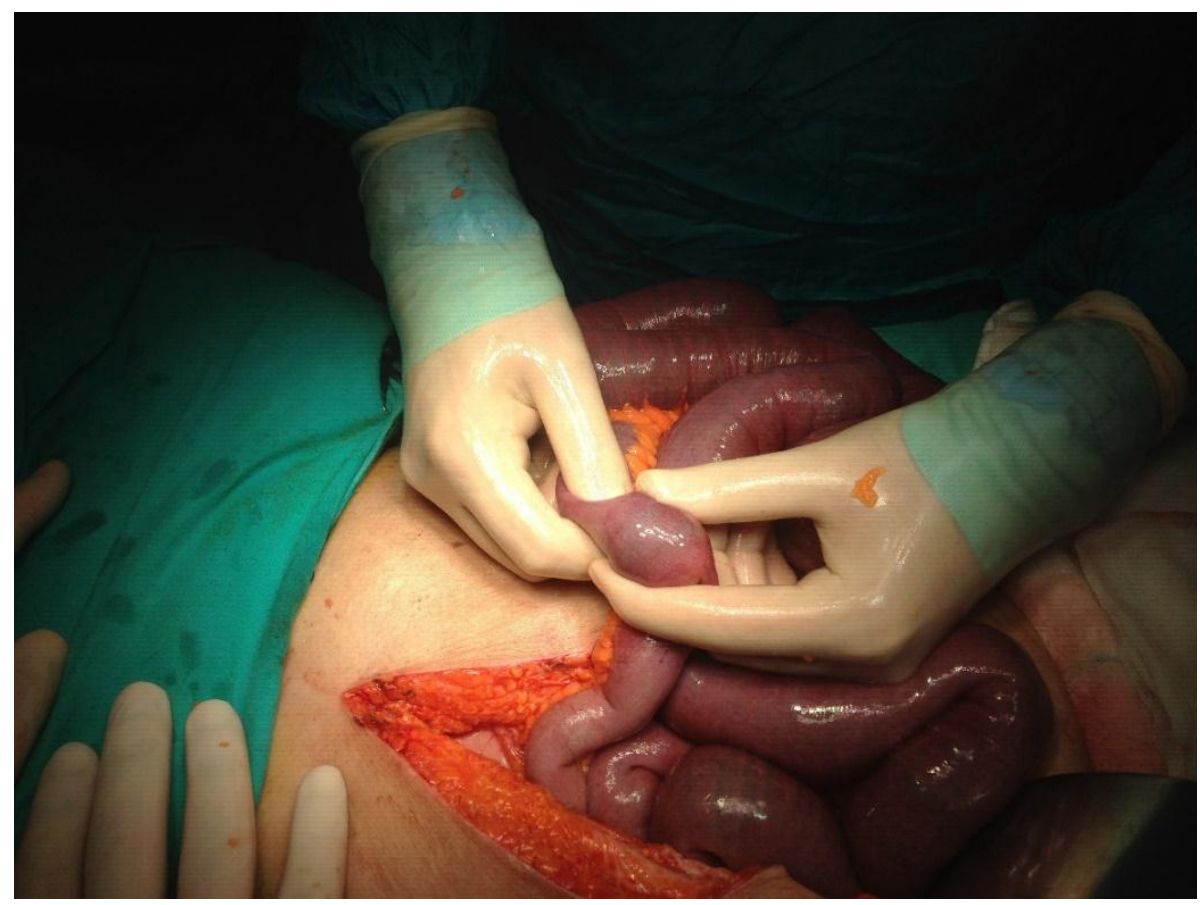

Figure $3.4 \mathrm{~cm}$ stone inside the intestinal lumen

The stone was removed via an enterotomy, and intestines were treated with primary closure (Figure 4). It was understood that the stone passed to the intestines via a cholecystoduodenal fistula. No other intestinal intraluminal stones were found in intraoperative assessment. The patient was followed up for 4 days after the operation with no problems and was discharged on postoperative 5 . day.

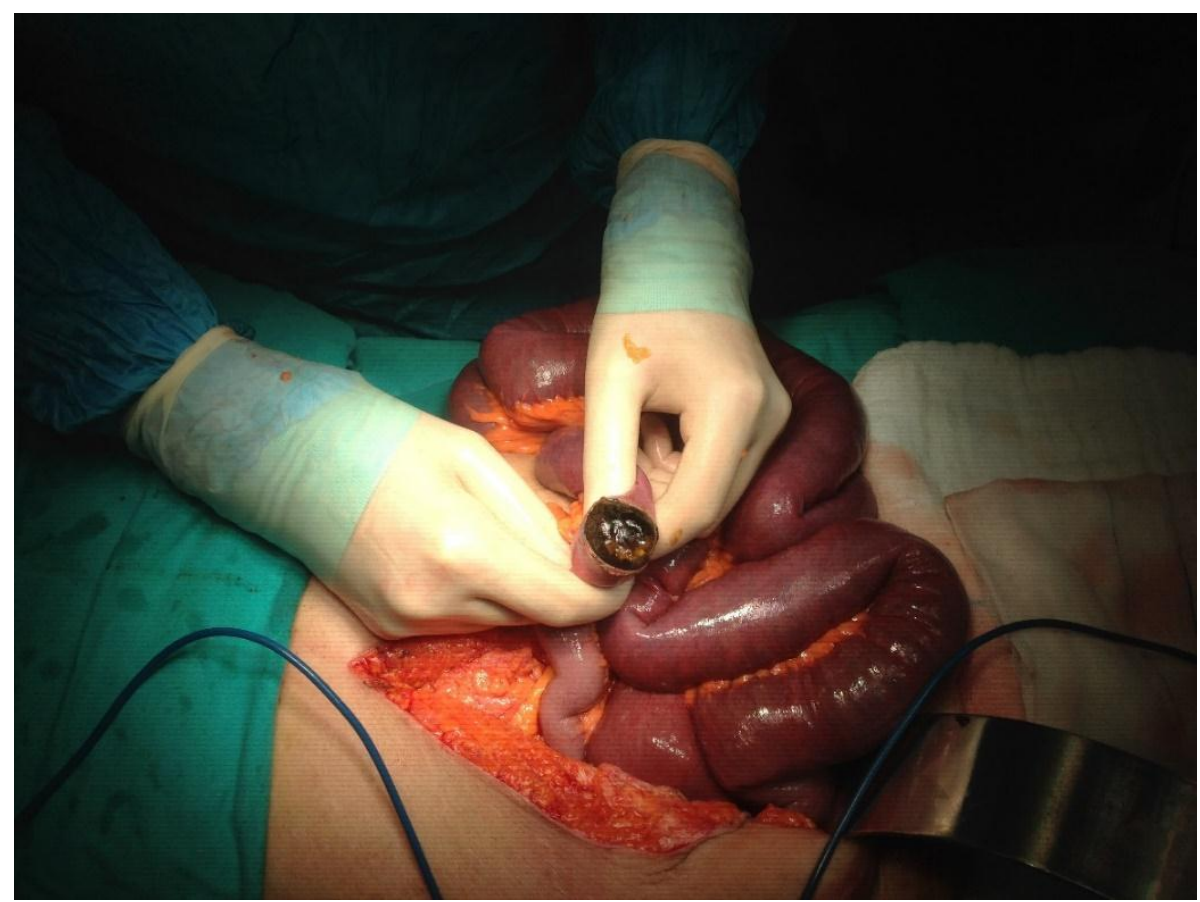

Figure 4. The removed stone via enterotomy

Most common gallstone complications are; acute and chronic cholecystitis, acute pancreatitis, choledocholithiasis and gangrenous gallbladder. Rare complications include; Mirizzi syndrome, cholecystoenteric fistula and ileus ${ }^{6}$. Gallstone ileus usually occurs through bilioenteric fistulas caused by gallstones bigger than $2.5 \mathrm{~cm}$ of diameter eroding and perforating the bladder wall. Cholecystoduodenal fistulas were detected in $68 \%$ of patients with gallstone ileus ${ }^{7}$. In our case of a 78 
year old female patient; the diameter of the stone which caused the obstruction was $4 \mathrm{~cm}$, and it was located in terminal ileum. Location of the cholecystoenteric fistula was cholecystoduodenal. Although it is present in the literature, pulverizing the stone and advancing smaller sized stones along the intestinal lumen and removing them is not a recommended treatment, due to intestines being edematic and fragile, and the possibility of being stuck in the ileocecal valve. This kind of treatment also causes trauma in fragile intestinal walls. Among the treatment options are enterolithotomy, single phased surgery (stone removal, cholecystectomy, fistula tract excision) and two phased surgery (enterolithotomy and biliary tract surgery). Laparoscopic diagnose and treatment is also an option ${ }^{8}$. Diagnosing gallstone ileus is difficult, both clinically and radiologically. $50 \%$ of the time, it is diagnosed during the operation ${ }^{7}$. Abdominal tomography is important in diagnosis. Findings of Rigler triad can be seen when examining the tomography carefully ${ }^{9}$. Balthazar and Schechter added; 2 air-fluid levels in the right upper quadrant and air findings in gallbladder and duodenal bulb to these findings ${ }^{10}$. In our case, gallstone ileus came first to mind due to; intestinal air-fluid levels in erect abdominal radiography, stone-like formation in abdomen ultrasonography, stone image which fills the entire lumen at ileal segmental level in computer tomography and previous examinations concordant to gallstones. We preferred only enterolithotomy in our case in order to not increase morbidity and mortality, based on data in literature.

Delaying diagnosis and treatment of gallstone ileus can cause some severe complications like electrolyte imbalance, ischemic lesions, intestinal ulcerations, abscess formations, perforation and peritonitis. In addition, coexisting diseases increase the risk of surgery in patients older than 65 years ${ }^{11}$. No complications occurred in the postoperative period for our patient.

In conclusion, although gallstone ileus is one of the rarer causes of intestinal obstruction, it should be kept in mind for older female patients with intestinal obstruction and a clinical history of gallbladder stone.

\section{REFERENCES}

1. Rodriguez JC, Casado F, Femandez MJ, Morales DJ, Naranjo A. Cholecystectomy and fistula closure versus enterolithotomy alone in gallstone ileus. Br J Surg 1997; 84: 634-7.

2. Richards WO, Williams LF. Obstruction of the large and small intestine. Surg Clin North Am 1988; 68: 355-76.

3. Way LW. Surgical diagnosis and treatment. Biliary tract. Current. 10th edition; Appleton and Lange 1994: 553-4.

4. Khaira HS, Thomas DR. Gallstone emesis and ileus caused by common hepatic duct-duodenal fistula. Br J Surg 1994; 81: 723.

5. Gümüş M, Gümüş H, Çelebi F. Safra Taşı İleusu. AÜTD 2002; 34: 47-9.

6. Newman HF, Northup JD, Rosenblum M, Abrams H. Complications of cholelithiasis. Am J Gastroenterol 1968; 50: 476-96.

7. Lobo DN, Jobling JC, Balfour TW. Gallstone ileus: diagnostic pitfalls and therapeutic successes. J Clin Gastroenterol 2000; 30: 72-6.

8. Clavien PA, Richon J, Burgan S, Rohner A. Gallstone ileus. Br J Surg. 1990; 77: 737-42.

9. Deitz DM, Standage BA, Pinson CW, McConnell DB, Krippaehne WW. Improving the outcome in gallstone ileus. Am J Surg 1986; 151: 572-6.

10. Balthazar EJ, Schechter LS. Air in Gallbladder: A Frequent Finding in Gallstone lleus. Am J Roentgenol 1978; 131: 219-22.

11. Reisner RM, Cohen JR. Gallstone ileus: a review of 1001 reported cases. Am Surg 1994; 60: 441-6. 